\title{
Black silicon - correlation between microstructure and Raman scattering
}

\author{
Stanislav Jurečka ${ }^{*}$, Emil Pinčík ${ }^{* *}$, Kentaro Imamura ${ }^{* * *}$, \\ Taketoshi Matsumoto $^{* * *}$, Hikaru Kobayashi ${ }^{* *}$
}

\begin{abstract}
Black silicon layers were formed on silicon substrate by the surface structure chemical transfer method and by anodic etching method. Properties of microstructure of formed layers were experimentally studied by the electron microscopy methods (TEM) and characterized by statistical, Fourier and multifractal methods. Theoretical structures with defined fractal properties and surface roughness were generated and their microstructure properties were evaluated. Obtained results were used for the explanation of the real structure development during the forming procedure. By using of this approach, we study the correlation of roughness and fractality with optical properties. Black silicon layers were also investigated by using of Raman scattering method. Optimized theoretical model describing the $1^{\text {st }}$ order of black Si Raman scattering profile was constructed and used for evaluation of the biaxial tensile stress introduced during etching procedure.
\end{abstract}

K e y w or d s: black silicon, TEM, roughness, fractal properties, Raman scattering

\section{Introduction}

For the solar cells low spectral reflectance is required for improving quality of prepared devices. Several methods of forming silicon surface (black silicon) were developed to reach this goal. Metal assisted wet chemical etching method has been used in producing features such as high aspect ratio nanowires [1-2], nanopores [3], tilted nanowires [4] and spirals [5]. Au and Ag metal particles were used in etching of $\mathrm{Si}$ in the presence of a solution of HF and oxidant. The metal catalyses the oxidation reaction that leads to Si etching. Black silicon structures can be also formed by reactive ion etching, stain etching, electrochemical etching, vapor chemical etching and other methods. An alternative method of forming porous $\mathrm{Si}$ structure is anodic etching. Anodic etching does not involve the use of an oxidant and does not require a contact metal to act as a catalyst. Applied electrical potential drives generated electric charges to the etchant/Si interface causing dissolution of Si substrate [6-7].

In the present study the nanocrystalline Si layers were formed by using the surface structure chemical transfer method (SSCT) [8-11]. In this procedure silicon surface is etched in solution of $\mathrm{H}_{2} \mathrm{O}_{2}$ and $\mathrm{HF}$. The Pt mesh roller contacted with the substrate acts as catalyst. Etching procedure is not sensitive to the crystallographic orientation of treated wafers and therefore structures with ultralow spectral reflectance can be formed on monocrystalline as well as multicrystalline $\mathrm{Si}$ wafers. The surface reaction rate is very high (nanostructured layer is formed only for several seconds). We studied properties of formed microstructures treated by different technological operations by using the transmission electron microscopy method (TEM). Experimental results were analysed by using Abbott-Firestone, statistical, Fourier and multifractal methods. The results of microstructure analysis were compared to results obtained by analysis of theoretically generated structures with defined roughness and fractal properties. By using of this approach, we study the interplay between roughness and fractality of studied structures.

Optical properties of black silicon layers formed by anodic etching procedure were also investigated by using of Raman scattering method. We developed efficient optimization method for construction of theoretical model describing the $1^{\text {st }}$ order Raman scattering profile. Resulting parameters were used in estimation of the biaxial tensile stress introduced by applied etching.

\section{Experimental details}

The nanostrucured layers were formed on cleaned flat $\mathrm{Si}$ surface by the surface structure chemical transfer method. The cross-sections of formed layers were scanned by the transmission electron microscope JEOL EM-3000F with the incident electron energy of $300 \mathrm{keV}$. A set of theoretical structures with different surface roughness and multifractal properties was numerically generated. Statistical and multifractal characteristics of these simulated

*Institute of Aurel Stodola, University of Žilina, Liptovský Mikuláš, Slovakia, stanislav.jurecka@feit.uniza.sk, **Institute of Physics SAS, Dúbravská cesta 9, 84228 Bratislava, Slovakia, ***The Institute of Scientific and Industrial Research, Osaka University, CREST, Japan Science and Technology Agency, Ibaraki, Osaka 567-0047, Japan

DOI: $10.2478 /$ jee-2019-0042, Print (till 2015) ISSN 1335-3632, On-line ISSN 1339-309X 

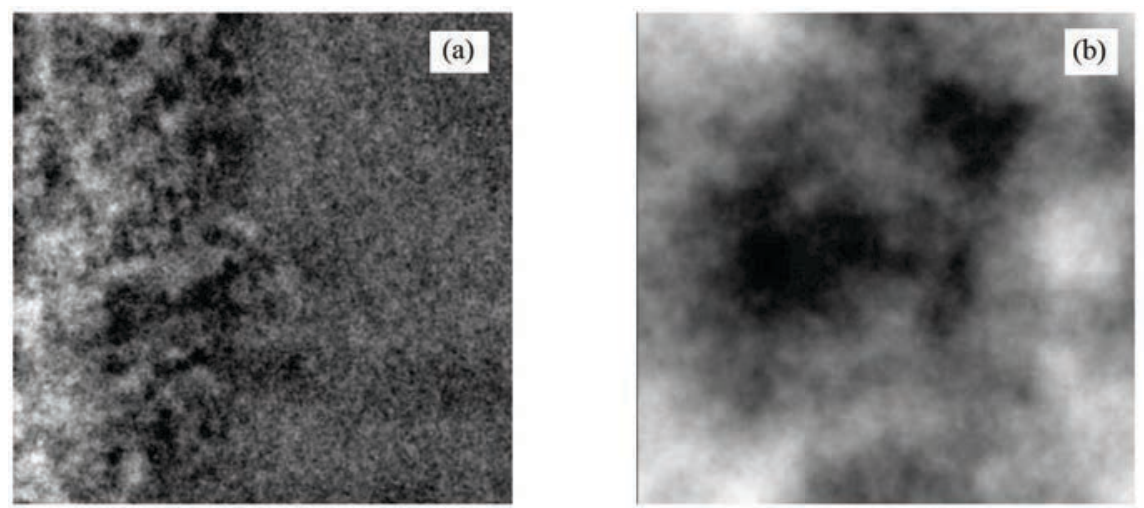

Fig. 1. (a) - TEM image of the SSCT nanostructure etched for 5 seconds, (b) $-h(x, y), r=10 \mathrm{~nm}, H=0.8$
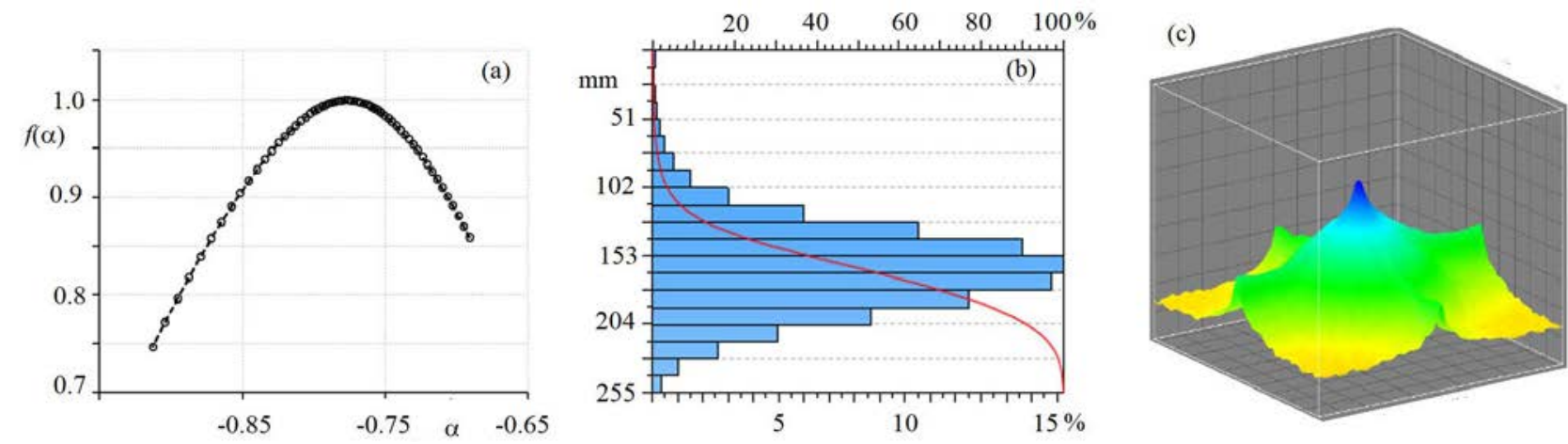

Fig. 2. Results of microstructure analysis: (a) - multifractal singularity spectrum $f(\alpha)$, (b) - AF curve and distribution, (c) - 2DFFT structure of the TEM image of the SSCT structure

structures as well as real nanostructured Si samples were analysed and compared. Porous black Si layers were also produced by anodic etching method. P-type Si substrates were cleaned by standard RCA procedure and etched for 300 seconds in HF under electrical potential $6.4 \mathrm{~V}$ and current $50 \mathrm{~mA}$. After etching samples were dried and annealed in oxygen atmosphere for 10 minutes at $850^{\circ} \mathrm{C}$. Raman scattering spectra were measured by using Thermo Scientific DXR Raman spectrometer with wavelength of laser excitation $\lambda=532 \mathrm{~nm}$.

\section{Results and discussion}

\subsection{Black silicon and theoretical fractal structures}

Black silicon layers formed by the SSCT method and porous layers formed by anodic etching show complex microstructures that cannot be properly described by means of conventional Euclidean geometry. The microstructure of formed layer is strongly influenced by etching conditions, especially by the etching time. In the SSCT structures with gradient of material density we observe several forms of symmetries connected with the dimensional scaling. The self-similarity of formed features and scaling properties are typical for fractal structures. Development of fractal characteristics of formed structure is closely related to individual steps of forming procedure
[12-14]. Information about the measured values observed at positions $(x, y)$ on the TEM image is in our approach implemented in the concept of the random height function $h(x, y)$. Experimentally determined $h(x, y)$ values from the TEM experiment were used for computation of the multifractal singularity spectra $f(\alpha)$ and generalized multifractal dimension $D q[15-16]$. In study of observed structures we numerically generated a series of theoretical surfaces by computation of the $h(x, y)$ functions with defined values of the RMS roughness $R q$ [17] and fractal Hölder coefficient $H$. Analysis of these theoretical structures enables estimation of the influence of the $R q$ and $H$ variation on the statistical, Fourier and multifractal properties of studied theoretical as well as real experimental layers. Generated area of images was $10 \times 10 \mu \mathrm{m}^{2}$ modelled by $512 \times 512$ image pixels. Area of $512 \times 512$ pixels at the TEM images was also selected in an evaluation of the statistical and fractal characteristics of the real structures.

The TEM image of the SSCT structure etched for 5 seconds is shown in Fig. 1a. Intensive modification of black Si layer in the volume of etched substrate can be clearly observed. In Fig. 1b surface constructed by random height function $h(x, y)$ with the RMS roughness $r=10 \mathrm{~nm}$ and fractal coefficient $H=0.8$ is shown. Structures observed on the TEM images and theoretical structures were analysed by multifractal [15], Abbott- 

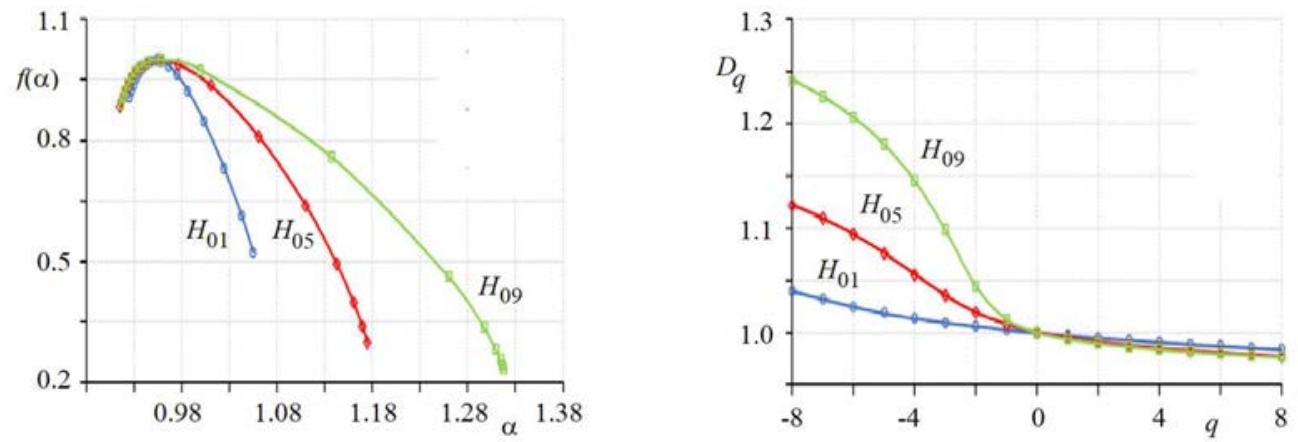

Fig. 3. Results of multifractal analysis of theoretical structures with different fractality
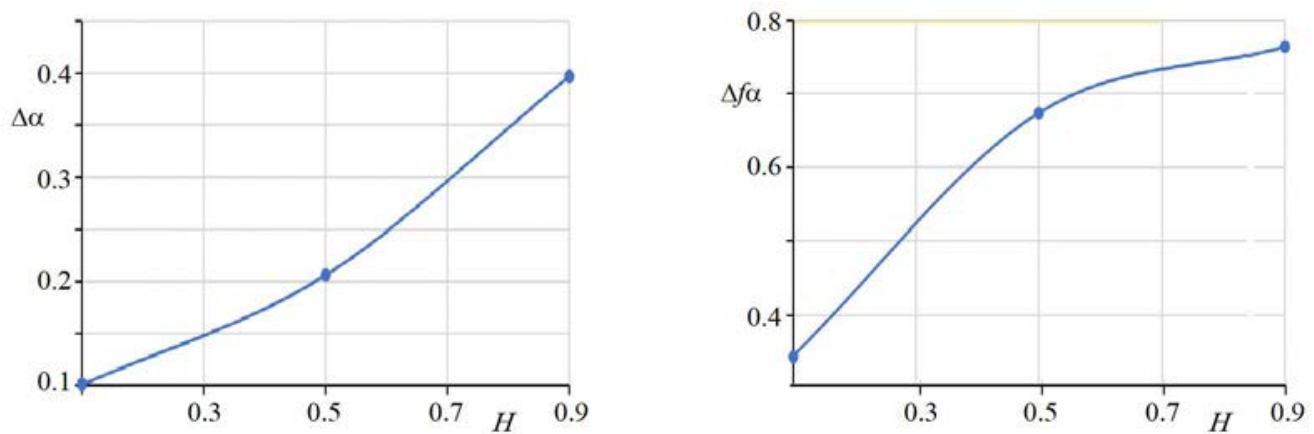

Fig. 4. Development of the multifractal functions $f(\alpha)$ and $D q$ with fractal exponent $H: \Delta \alpha, \Delta f(\alpha)$
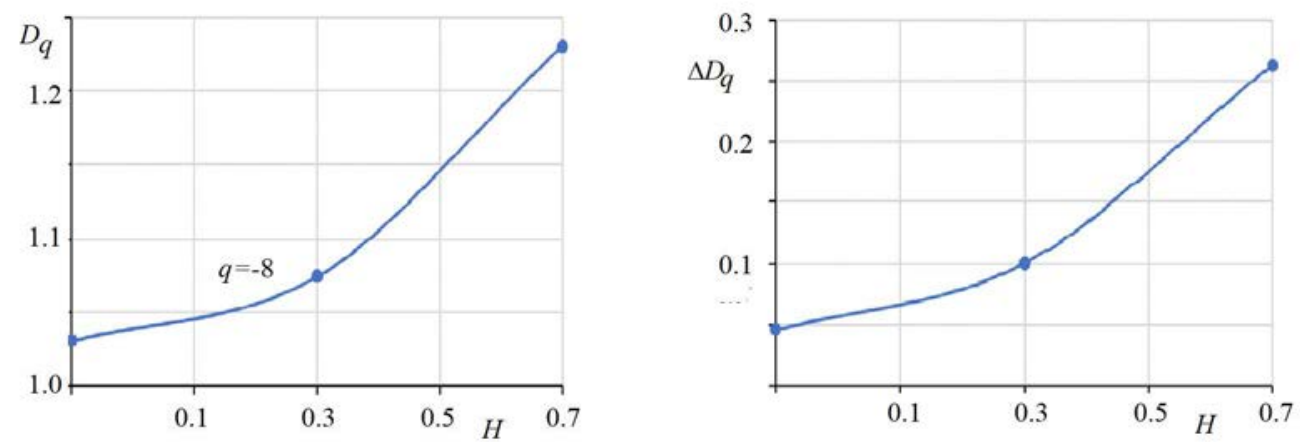

Fig. 5. Development of the multifractal functions $f(\alpha)$ and $D q$ with fractal exponent $H: D q, q=-8$ and $\Delta D q$
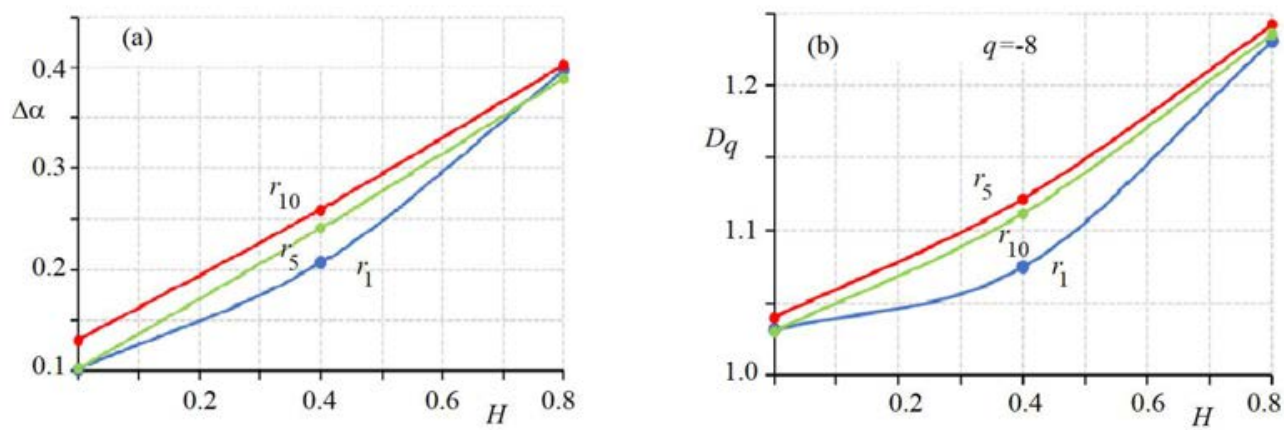

Fig. 6. Development of the multifractal functions $f(\alpha)$ and $D q$ with the RMS roughness $r: \Delta \alpha, D q, q=-8$ 

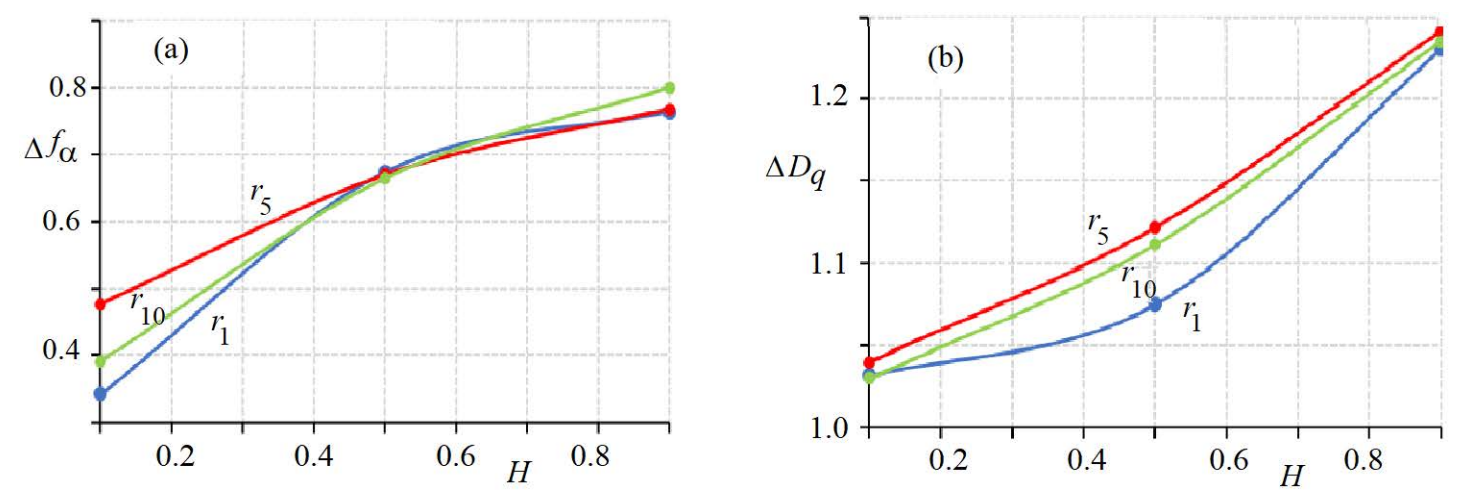

Fig. 7. Development of the multifractal functions $f(\alpha)$ and $D q$ with the RMS roughness $r: \Delta f(\alpha)$ and $\Delta D q$
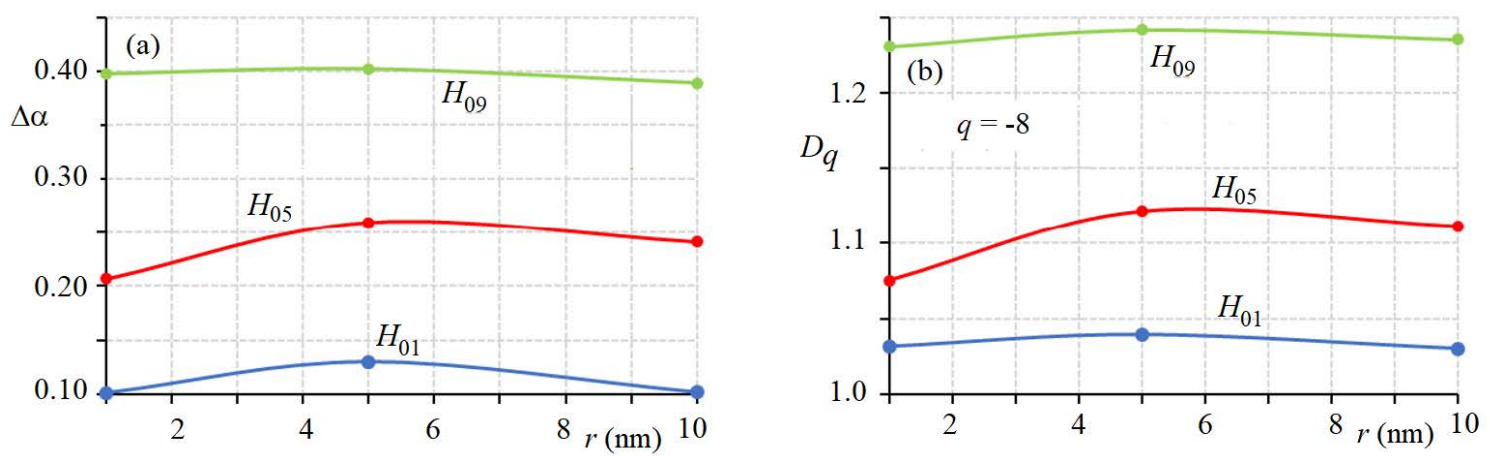

Fig. 8. Development of the multifractal functions $f(\alpha)$ and $D q$ with fractal coefficient $H: \Delta \alpha, D q, q=-8$
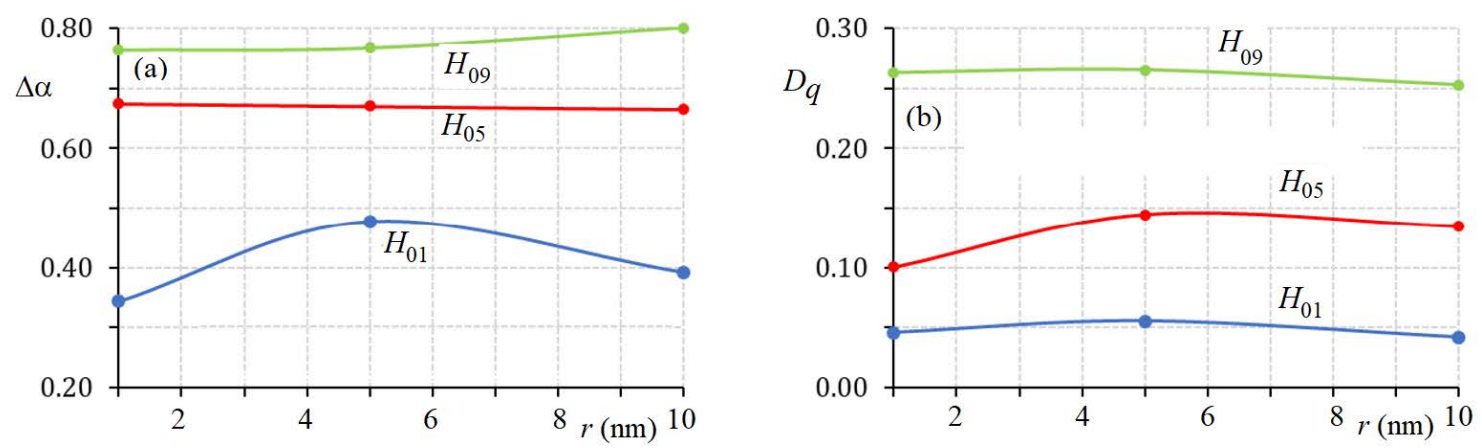

Fig. 9. Development of the multifractal functions $f(\alpha)$ and $D q$ with fractal coefficient $H: \Delta f(\alpha)$ and $\Delta D q$

Firestone (AF) [18], and Fourier (2D FFT transformation [19]) methods. Multifractal singularity spectrum $f(\alpha)$ of the SSCT structure is shown in Fig. 2a. The concave shape of $f(\alpha)$ curve is significant for multifractal structure. We studied influence of fractal coefficient $H$ and RMS roughness values on the microstructure properties on a set of simulated random height functions. Properties of multifractal singularity spectrum $f(\alpha)$, generalized fractal dimension $D q$, histograms of $h(x, y)$ values, and AF curves are shown in Fig. 2-11.

In Fig. $2 \mathrm{~b}$ the Abbott-Firestone curve (AF) is shown. The shape of the AF curve provides information about distribution of peaks and valleys observed in the structure. Above the highest value of the $h(x, y)$ function $0 \%$ bearing area is observed and under the deepest valley $100 \%$ bearing area is obtained. The AF curves of theoretical $h(x, y)$ structures provide in comparison to the real structure information about the influence of roughness and fractality on the microstructure properties (see Fig. 11).

In Fig. 2c the structure of Fourier domain of the SSCT sample is shown. It is closely connected with the distribution of small features in observed SSCT image and provide information related to the random height function values, studied by statistical methods (see histograms in Fig. 10, 11). In Fig. 3 multifractal singularity spectrum $f(\alpha)$ and generalized fractal dimension $D q$ determined for theoretical structure with the RMS roughness $r=5$ 

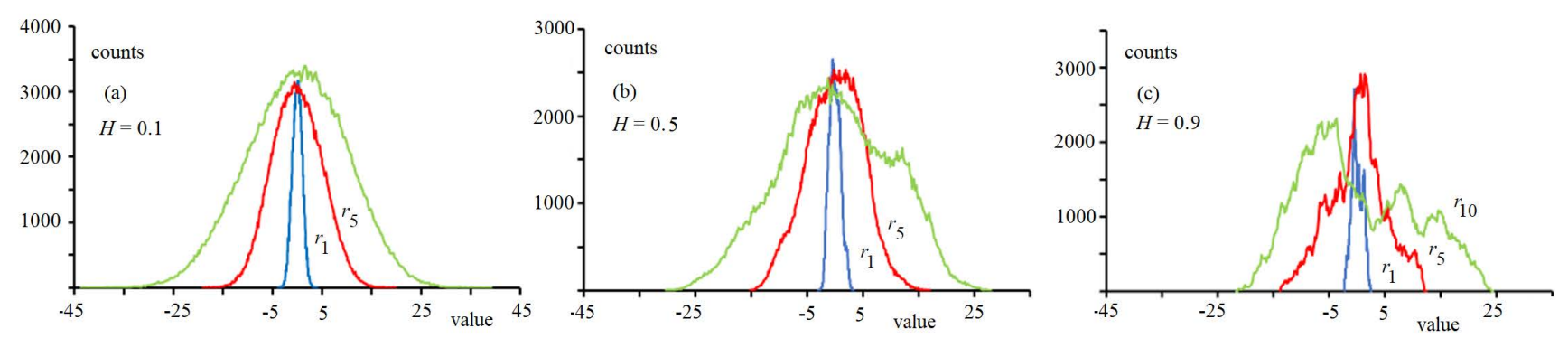

Fig. 10. Influence of RMS roughness: distributions of the $h(x, y)$ values for structures with the fractal coefficient $H=0.1,0.5$ and 0.9
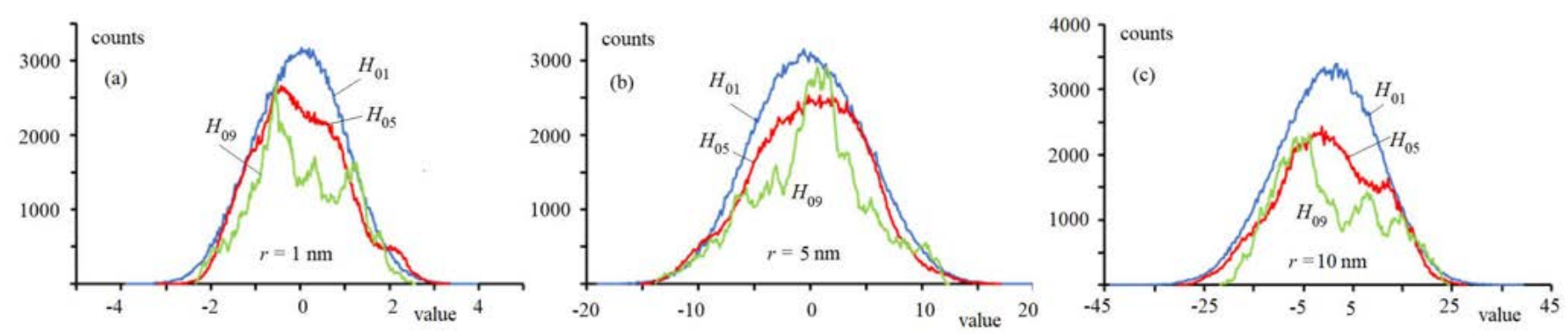

Fig. 11. Influence of fractality: distributions of the $h(x, y)$ values for structures with the RMS roughness of $r=1,5$ and $10 \mathrm{~nm}$

$\mathrm{nm}$ are shown. Fractal coefficient was set to $H=0.1$, 0.5 and 0.9 . Obtained results illustrate strong influence of the $H$ value on the multifractal analysis results $f(\alpha)$ and $D q$.

From the $f(\alpha)$ and $D q$ curves we determined following parameters: $\Delta \alpha, \Delta f(\alpha), \Delta D q$ and development of the $D q$ value for $q=-8,(D q, q=-8)$. Obtained results are shown in Fig. 4 and Fig. 5. These parameters illustrate the influence of the fractality represented by changes of $H$ value on the structure with relatively small RMS roughness of $r=5 \mathrm{~nm}$.

We constructed theoretical surfaces with different RMS roughness $r=1,5$ and $10 \mathrm{~nm}$ and different fractal coefficient values $H=0.1,0.5$ and 0.9 . Results of multifractal analysis of these surfaces are shown in figures Fig. 6-9.

The influence of RMS roughness on the distribution of the $h(x, y)$ values for structures with the fractal coefficient $H=0.1,0.5$ and 0.9 is illustrated in Fig. 10 and the influence of fractality is shown in Fig. 11 for structures with RMS roughness $r=1,5$ and $10 \mathrm{~nm}$.

In all cases, for structures with small as well as with higher roughness, we observe dominant role of the fractal coefficient $H$. With increasing of fractal $H$ value, the amplitude of distribution of random height function is modified very intensively for all values of selected RMS roughness (see Fig. 11). Changes of the RMS roughness modify dominantly the width of the distribution (see Fig. $10)$.

Results of the Abbott-Firestone analysis of theoretically generated structures $h(x, y)$ are shown in Fig. 12 .
Fractal coefficient values $H$ are not significant for the shape of AF curve (Fig. 12a). On the other hand, the RMS roughness influences the distribution of peaks and valleys significantly and with increasing RMS roughness the modification of AF curve is emphasized (Fig. 12b).

Microstructure of the SSCT layers show dominant multifractal properties modified by the roughness. It is caused by very high speed of etching resulting in forming of structure with the particle size gradient. Self-similarity property of formed particles causes importance of fractal properties of resulting structure. This kind of structure show very low spectral reflectance in wide range of wavelengths [8-11].

\subsection{Optical properties of anodic etched silicon layers}

We fabricated black Si structures also by anodic etching method. Properties of formed porous structures were experimentally studied by Raman scattering. Theoretical model of the $1^{\text {st }}$ order Raman scattering peak of black silicon structure was constructed by implementing of pseudo-Voigt function for the Si scattering profile. Pseudo-Voight function [20-21] is in our approach defined by equation

$$
\begin{gathered}
I_{\mathrm{pV}}(\nu)=I_{\max }\left[\mu \frac{2}{\pi} \frac{w}{4\left(\nu-\nu_{\mathrm{c}}\right)^{2}+w^{2}}\right]+ \\
I_{\max }\left[(1-\mu) \frac{\sqrt{4 \ln 2}}{w \sqrt{\pi}} \exp \left\{-\frac{4 \ln 2}{w^{2}}\left(\nu-\nu_{\mathrm{c}}\right)^{2}\right\}\right] .
\end{gathered}
$$

Parameter $\mu$ is used for definition of Gaussian and Lorentzian content in the pseudo-Voigt profile, $\nu_{\mathrm{c}}$ is cen- 

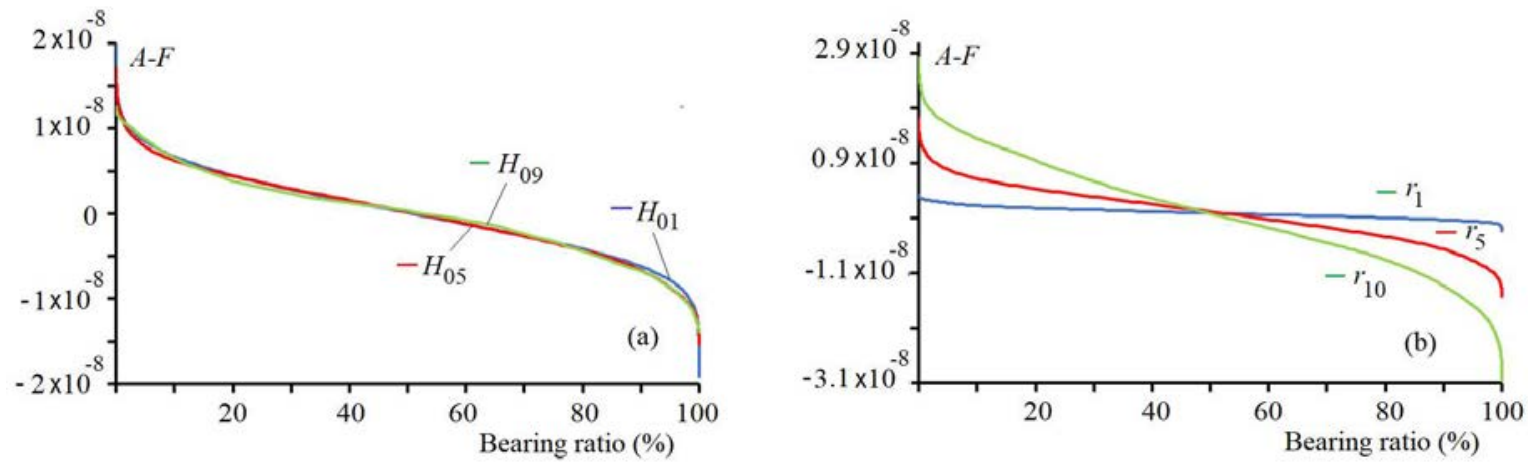

Fig. 12. Results of the Abbott-Firestone analysis: (a) - influence of the fractal coefficient $H$, and (b) - influence of the RMS roughness
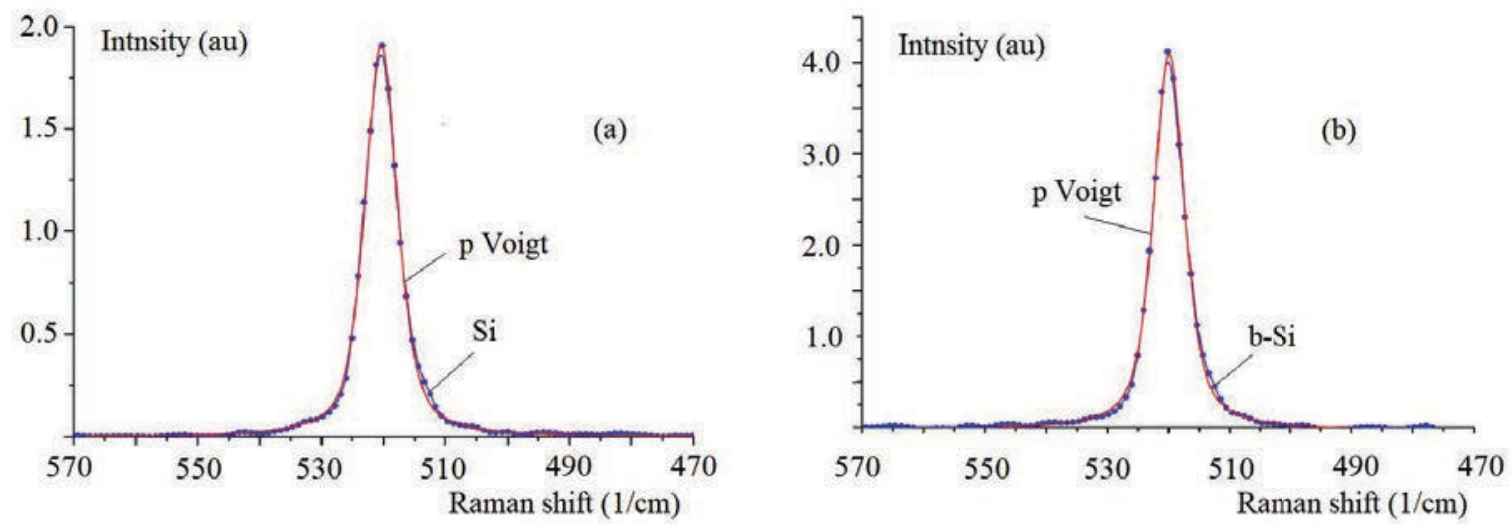

Fig. 13. Experimental data and theoretical models of Raman scattering for (a) - Si, and (b) - anodic etched black Si constructed by the pseudo-Voigt model function

tre of Raman peak and $w$ is the thickness of modelled profile. Theoretical model of $I_{\mathrm{pV}}$ was constructed in two steps. In the first step initial values if the model function were estimated in interactive graphical environment. Estimated initial values were then refined in optimization step based on implementation of stochastic optimization procedure (genetic algorithm) combined with LevenbergMarquardt optimization algorithm [22-24]. These optimization steps provide information for reliable determination of the $1^{\text {st }}$ order Raman scattering profile and study of the crystal lattice distortions. Refined values of theoretical pseudo-Voigt profile model of the Raman scattering of black Si structure are given in Table 1. Resulting theoretical models for the Si substrate and black Si structure formed by anodic etching method are shown in Fig. 13a and Fig. 13b, respective.

Table 1. Parameters of the pseudo-Voigt profile functions of the theoretical model of the $1^{\text {st }}$ order Raman scattering

\begin{tabular}{lcc} 
& $\mathrm{Si}$ & black Si \\
\hline$\nu_{\mathrm{c}}$ & 520.361 & 519.923 \\
$I_{\max }$ & 16281.708 & 34328.319 \\
$w$ & 6.248 & 6.055 \\
$\mu$ & 0.679 & 0.7005 \\
\hline
\end{tabular}

Center $\nu_{\mathrm{c}}$ of Raman profile from black $\mathrm{Si}$ is shifted towards lower wavenumbers. This is caused by the deformations of Si lattice structure during etching and results in introduction of tensile biaxial stress. Value $\nu_{\mathrm{c}}(\mathrm{Si})$ $\nu_{\mathrm{c}}($ black $\mathrm{Si})=0.438 \mathrm{~cm}^{-1}$ corresponds to isotropic biaxial tensile stress of $\left(\sigma_{\mathrm{xx}}+\sigma_{\mathrm{yy}}\right)=228 \mathrm{MPa}$ introduced by the anodic etching procedure.

\section{Conclusions}

Black Si structures were formed by the SSCT and anodic etching methods. Formed SSCT microstructure with gradient of particle size show characteristic multifractal properties. In this work the role of fractal coefficient $H$ and RMS roughness were studied theoretically. We discovered dominant role of fractality in forming properties of structures with various RMS roughnesses and dominant role of RMS roughness influencing distribution of peaks and valleys as well as modifying the width of the distribution of random height function values. We also discovered the shift of the position of the $1^{\text {st }}$ order Raman scattering profile in anodic etched silicon structure. This effect is caused by isotropic biaxial tensile stress introduced by the anodic etching procedure. 


\section{Acknowledgements}

The work was supported by project H2020-MSCARISE-2016-6260922, grant of Science and Technology Assistance Agency APVV-15-0152, Scientific Grant Agency of the Ministry of Education of Slovak Republic and the Slovak Academy of Sciences project VEGA 1/0676/17, VEGA 2/0149/18, Centre of Excellence of Power Electronics Systems and Materials ITMS 26220120003, No. OPVaV-2008/2.1/01-SORO, ITMS 26220120046, and by project ITMS 26210120021, co-funded from EU sources and European Regional Development Fund.

\section{REFERENCES}

[1] S.-W. Chang, V. P. Chuang, S. T. Boles, C. A. Ross, and C. V. Thompson, "Densely Packed Arrays of Ultra-High-Aspect-Ratio Silicon Nanowires Fabricated using Block-Copolymer Lithography and Metal-Assisted Etching", Advanced Functional Materials, vol. 19, pp. 2495-2500, 2009.

[2] M. L. Zhang, K. Q. Peng, X. Fan, J. S. Jie, R. Q. Zhang, S. T. Lee, and N. B. Wong, "Preparation of Large-Area Uniform Silicon Nanowires Arrays through Metal-Assisted Chemical Etching", J. Phys. Chem. C, vol. 112, pp. 4444-4450, 2008.

[3] X. Li, Y. Xiao, C. Yan, K. Zhou, S. L. Schweizer, A. Sprafke, J.-H. Lee, and R. B. Wehrspohn, "Influence of the mobility of Pt nanoparticles on the anisotropic etching properties of silicon", ECS Solid State Lett., vol. 2, pp. 22-24, 2013.

[4] H. Chen, H. Wang, X.-H. Zhang, C.-S. Lee, and S.-T. Lee, "Wafer-Scale Synthesis of Single-Crystal Zigzag Silicon Nanowire Arrays with Controlled Turning Angles", Nano letters, vol. 10, pp. 864-868, 2010.

[5] O. J. Hildreth, A. G. Fedorov, and C. P. Wong, "3D spirals with controlled chirality fabricated using metal-assisted chemical etching of silicon", ACS Nano, vol. 6, pp. 10004-10012, 2012.

[6] L. T., Canham, "Silicon Quantum Wire Array Fabrication by Electrochemical and Chemical Dissolution of Wafers", Applied Physics Letters, vol. 57, pp. 1046-1048, 1990.

[7] V. Lehmann, and U. Gösele, "Porous silicon formation: A quantum wire effect", Applied Physics Letters, vol. 58, pp. 856-858, 1991.

[8] K. Imamura, T. Nonaka, D. Irishika and H. Kobayashi, Ecs Solid State Lett., vol. 4, pp. Q63-Q65, 2015.
[9] D. Irishika, K. Imamura and H. Kobayashi, Sol. Energ. Mat. Sol. Cells, vol. 141, 2015.

[10] K. Imamura, D. Irishika, and H. Kobayashi, Prog. Photovolt. Res. Appl., vol. 25, pp. 358-366, 2017.

[11] K. Imamura, F. C. Franco Jr., T. Matsumoto, H. Kobayashi, Appl. Phys. Lett., vol. 103, pp. 013110, 2013.

[12] B. B. Mandelbrot, The Fractal Geometry of Nature, Freeman: New York, USA, 1983.

[13] K. Falconer, Fractal Geometry, Wiley: Chichester, UK, 1989.

[14] G. Franceschetti, D. Riccio, Scattering, Natural Surfaces and Fractals, Academic Press: Burlington, USA, 2007.

[15] A. Chhabra, R. V. Jensen, Phys. Rew. A, vol. 40, pp. 5284-5294, 1989.

[16] E. A. F. Ihlen, B. Vereijken, "Interaction dominant dynamics in human cognition: beyond 1/f fluctuations", J. Exp. Psychol. Gen., vol. 139, pp. 436-463, 2010.

[17] American Standard ASME B46.1 for Surface Texture, 1995.

[18] J. Schmahling, F. A. Hamprecht, "Generalizing the AbbottFirestone curve by two new surface descriptors", Wear, vol. 262, pp. 1360-1371, 2007.

[19] W. Smith, Handbook of Real-Time Fast Fourier Transforms: Algorithms to Product Testing, Wiley, IEEE Press, 1995.

20] V. Jain, M. C. Biesinger, M. R. Linford, "The GaussianLorentzian Sum, Product, and Convolution (Voigt) functions in the context of peak fitting X-ray photoelectron spectroscopy (XPS) narrow scans", Applied Surface Science, vol. 447, pp. 548-553, 2018.

[21] P. Pelikán, M. Čeppan, M. Liška, Applications of Numerical Methods in Molecular Spectroscopy, CRC Press, Boca Raton, 1993.

[22] S. Jurečka, "Theoretical Model of the Physical System: Optimization by the Genetic Algorithm", In: Ioannis Dritsas Stochastic Optimization - Seeing the Optimal for the Uncertain, InTech, Vienna, 2011.

[23] S. Jurečka, E. Pinčík, R., Brunner, "Solution of the optical parameters of the thin film systems and interfaces", Applied Surface Science, vol. 254, pp. 3672-3676, 2008.

[24] L. S. Lasdon, A. D. Waren, A. Jain, M. Ratner, "Design and Testing of a Generalized Reduced Gradient Code for Nonlinear Programming", ACM Transactions on Mathematical Software, vol. 4, no. 1, pp. 34-50, 1978.

Received 19 March 2019 